\title{
Erratum to: Tumors of Uncertain Differentiation
}

\author{
Simon David Sprengel, Marc-André Weber, \\ Hendrik R. Degryse, and Filip M. Vanhoenacker
}

\section{Erratum to}

Chapter 18 in F.M. Vanhoenacker et al. (eds.), Imaging of Soft Tissue Tumors, DOI 10.1007/978-3-319-46679-8_18

An error in the production process unfortunately led to publication of this chapter prematurely, before incorporation of the final corrections. The version supplied here has been corrected and approved by the author.

The updated online version of this chapter can be found at DOI 10.1007/978-3-319-46679-8_18 